\title{
Optimal Static Pricing for a Tree Network*
}

\author{
Felipe Caro $^{\dagger}$ \\ David Simchi-Levi ${ }^{\ddagger}$ \\ February 1, 2012
}

Forthcoming in Annals of Operations Research DOI: 10.1007/s10479-012-1115-4

The final publication is available at www.springerlink.com

\begin{abstract}
We study the static pricing problem for a network service provider in a loss system with a tree structure. In the network, multiple classes share a common inbound link and then have dedicated outbound links. The motivation is from a company that sells phone cards and needs to price calls to different destinations. We characterize the optimal static prices in order to maximize the steady-state revenue. We report new structural findings as well as alternative proofs for some known results. We compare the optimal static prices versus prices that are asymptotically optimal, and through a set of illustrative numerical examples we show that in certain cases the loss in revenue can be significant. Finally, we show that static prices obtained using the reduced load approximation of the blocking probabilities can be easily obtained and have near-optimal performance, which makes them more attractive for applications.
\end{abstract}

\section{Introduction}

We consider a network service provider that can be modelled as a loss system with a tree topology. Multiple customer classes share a common link and each class has its own outbound (or inbound) link, as shown in Figure 1. The classes are characterized by a price-dependent arrival rate and the random time they remain in the system. All classes are assumed to have the same bandwidth requirement. This can be seen as the case when all classes demand the same service level which can be satisfied by allocating a constant amount of resource in the corresponding links. Though many situations could fit in this framework, for example a dial-up Internet provider or a call center, this problem was originally thought for a company that sells phone cards. In that case, the customer classes represent call destinations, for example, the phone calls to Santiago, Chile on a given day.

\footnotetext{
* Research supported in part by the Center of eBusiness at MIT, ONR Contracts N00014-95-1-0232 and N0001401-1-0146, and by NSF Contracts DMI-9732795, DMI-0085683 and DMI-0245352.

${ }^{\dagger}$ UCLA Anderson School of Management, 110 Westwood Plaza Suite B-420, Los Angeles, CA 90095, USA. email: fcaro@anderson.ucla.edu

${ }^{\ddagger}$ Dept. of Civil and Environmental Engineering and the Engineering Systems Division, MIT, 77 Massachusetts Ave. Rm. 1-171 Cambridge, MA 02139, USA. email: dslevi@mit.edu
} 


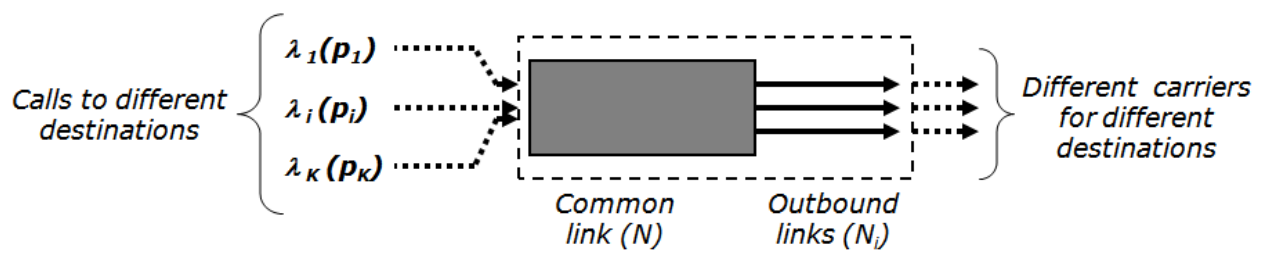

Figure 1: A loss system with a tree topology.

Our objective is to characterize the optimal static prices in order to maximize the provider's revenue. We focus on static prices since this is the current standard in the phone card setting. Indeed, instantaneous changes in prices are not well received among customers and can cause goodwill losses for the company. Moreover, our results confirm that the loss in revenue from using static over dynamic prices is usually small.

There is a vast literature on loss systems. Most of the effort has concentrated on finding structural properties for the blocking probabilities and studying capacity allocation policies (see Kelly [6] and Ross [15]). Though pricing in queueing models with infinite buffers has a long history - see Mendelson [11] for one of the first seminal papers and see Courcoubetis and Weber [4] for more recent references-, the same topic in loss systems has received much less attention. The exception are models for a single link, which includes Courcoubetis and Reiman [3], Lanning et al. [8], and Carrizosa et al. [2].

For our purposes, Ziya et al. [21] and Paschalidis and Tsitsiklis [12] are the most relevant references in the single-link pricing literature. Ziya et al. consider multiple classes and show how to reduce the pricing decisions to a single-dimension optimization problem. Then they use the new formulation to establish analytical results. One of their findings is that the optimal prices are decreasing in the capacity of the common link. Interestingly, we show the same result in this paper using an alternative single-dimension reduction (note that both reductions were derived independently). On the other hand, Paschalidis and Tsitsiklis consider a dynamic pricing model and they show that static prices are asymptotically optimal in the sense that they achieve a performance upper bound when the number of users grows to infinity in unison with the system's capacity. The extension of the asymptotic result to the network setting, in particular, a tree network, is done by Paschalidis and Liu [13]. They characterize the rate of convergence to the asymptotic regime and show that the analysis also carries through when substitution is allowed. Finally, with a slightly different approach, Lin and Shroff [9] show that the static prices induced by the upper bound are themselves asymptotically optimal. We take these prices as a benchmark and show that the revenue loss with respect to the optimal static prices outside the asymptotic regime can be significant in some cases.

In this paper we specifically study the pricing problem for a tree network, which includes the single-link as a special case. Though the latter is shown to be a well-behaved problem, a general tree network is significantly more challenging and properties such as monotonicity and quasiconcavity cannot be taken for granted. In terms of contributions, from an analytical standpoint, we provide new structural findings (Propositions $1 \& 2$ and Lemmas $2 \& 3$ ) as well as short alternative proofs 
to established results (Lemma 1 and Propositions $3 \& 4$ ). From a practical point of view, we show that static prices computed using the reduced load approximation of the blocking probabilities have near optimal performance. Overall, we derive insights that help to identify when an exact or an asymptotic approach for pricing optimization is more desirable.

The remainder of the paper has the following structure: The next section $\S 2$ introduces the tree network topology and the static pricing model. The characterization of the optimal static prices is presented in $\S 3$. The asymptotically optimal prices are introduced in $\S 4$ and the numerical analysis is described in $\S 5$. Finally, $\S 6$ summarizes the conclusions and possible extensions to more general loss networks.

\section{The Model}

In what follows, boldface symbols represent vectors. Let $k \in \mathcal{K}=\{1, \ldots, K\}$ be the different call destinations. The common link has capacity $N$ and represents the physical switch where all incoming calls are received. Each outbound link has capacity $N_{k}$ and represents a carrier for a particular destination. Let $\mathbf{C}=\left(N, N_{1}, \ldots, N_{K}\right)$ denote the capacity vector. We assume $\mathbf{C}$ to be given, which is usually the case at a tactical level.

Let $p_{k} \in\left[0, p_{k}^{\max }\right]$ be the price per unit of time charged to class- $k$ customers. Class- $k$ customers arrive to the system according to an independent Poisson process with rate $\lambda_{k}\left(p_{k}\right)$ and average holding time $1 / \mu_{k}$. We do not consider substitution effects among classes. This is reasonable in the case of a phone card company (variations in the price for calls to China does not affect the demand for calls to Chile). The functions $\lambda_{k}\left(p_{k}\right)$ are assumed to be continuously differentiable and strictly decreasing with inverse $p_{k}\left(\lambda_{k}\right)$. Let $R_{k}\left(\lambda_{k}\right) \equiv \lambda_{k} \cdot p_{k}\left(\lambda_{k}\right)$ be the instantaneous reward rate, with $R_{k}^{\prime}(0)>0, \forall k \in \mathcal{K}$, to exclude trivial solutions. Let $y_{k}=\lambda_{k} / \mu_{k}$ be the offered load of class $k$ and let $\mathbf{y}$ be the corresponding vector. In the analysis we let the service provider decide the arrival rate $\boldsymbol{\lambda}$ or the offered load $\mathbf{y}$, which in turn determine the price $\mathbf{p}$.

An incoming class- $k$ call is admitted as long as there is at least one line available in the common link and in the $k$-th dedicated link. Otherwise, the called is blocked and lost. For class $k$, let $X_{k}(\boldsymbol{\lambda} ; \mathbf{C})$ be the number of calls in steady-state and let $\bar{B}_{k}(\boldsymbol{\lambda} ; \mathbf{C})$ be the nonblocking probability. Let $\Lambda=\left[0, \lambda_{1}^{\max }\right] \times \cdots \times\left[0, \lambda_{K}^{\max }\right]$, where $\lambda_{k}^{\max }<\lambda_{k}(0), \forall k \in \mathcal{K}$. The objective of the network service provider is to maximize the steady-state revenue so it solves

$$
\max _{\boldsymbol{\lambda} \in \Lambda} J(\boldsymbol{\lambda} ; \mathbf{C}) \equiv \sum_{k=1}^{K} p_{k}\left(\lambda_{k}\right) \mathbb{E}\left[X_{k}(\boldsymbol{\lambda} ; \mathbf{C})\right]=\sum_{k=1}^{K} \frac{R_{k}\left(\lambda_{k}\right)}{\mu_{k}} \bar{B}_{k}(\boldsymbol{\lambda} ; \mathbf{C}),
$$

where the last equality follows from Little's Law. Note that with an appropriate definition of the $R_{k}\left(\lambda_{k}\right)$ functions the model could also fit a social welfare maximization problem such as the consumer surplus model proposed by Paschalidis and Tsitsiklis [12].

Given the capacity vector $\mathbf{C}$, let $\Omega(\mathbf{C})=\left\{\mathbf{n} \in \mathbb{N}^{K} \mid n_{k} \leq N_{k}, \forall k \in \mathcal{K}\right.$, and $\left.\sum_{k=1}^{K} n_{k} \leq N\right\}$ be the feasible state space and let $\mathbf{b}_{k} \equiv \mathbf{e}_{1}+\mathbf{e}_{k+1}$, where $\mathbf{e}_{i}$ is the $i$-th canonical unit vector in $\mathbb{R}^{K+1}$. For the non-blocking probabilities, we can write $\bar{B}_{k}(\boldsymbol{\lambda} ; \mathbf{C})=G\left(\boldsymbol{\lambda} ; \mathbf{C}-\mathbf{b}_{k}\right) / G(\boldsymbol{\lambda} ; \mathbf{C})$ where $G(\boldsymbol{\lambda} ; \mathbf{C})=\sum_{\mathbf{n} \in \Omega(\mathbf{C})} \prod_{k=1}^{K} \frac{\left(\lambda_{k} / \mu_{k}\right)^{n_{k}}}{n_{k} !}$ is the product-form normalization constant for loss systems (see $[6,15])$. The identity $\frac{\partial G(\boldsymbol{\lambda} ; \mathbf{C})}{\partial \lambda_{k}}=G\left(\boldsymbol{\lambda} ; \mathbf{C}-\mathbf{b}_{k}\right) / \mu_{k}$ is used repeatedly in the analysis below. 
Directly differentiating the revenue function in Equation (1) with respect to $\lambda_{k}$, and assuming $N_{k}>0$ so that $\frac{\partial G}{\partial \lambda_{k}} \neq 0$, we obtain:

$$
\frac{\partial J(\boldsymbol{\lambda} ; \mathbf{C})}{\partial \lambda_{k}}=\frac{\bar{B}_{k}(\boldsymbol{\lambda} ; \mathbf{C})}{\mu_{k}}\left[R_{k}^{\prime}\left(\lambda_{k}\right)-\left(J(\boldsymbol{\lambda} ; \mathbf{C})-J\left(\boldsymbol{\lambda} ; \mathbf{C}-\mathbf{b}_{k}\right)\right)\right] .
$$

From the formula above, the first-order conditions for the optimal static arrival rates $\boldsymbol{\lambda}^{*}$ can be expressed as follows:

$$
R_{k}^{\prime}\left(\lambda_{k}^{*}\right)=J\left(\boldsymbol{\lambda}^{*} ; \mathbf{C}\right)-J\left(\boldsymbol{\lambda}^{*} ; \mathbf{C}-\mathbf{b}_{k}\right) \forall k \text { such that } \lambda_{k}^{*}>0 .
$$

The condition says that at the optimum, if class $k$ is active (i.e. $\lambda_{k}^{*}>0$ ), then the marginal revenue of class $k, R_{k}^{\prime}\left(\lambda_{k}^{*}\right)$, must be equal to the opportunity cost of admitting a call of class $k$ and therefore blocking future calls of other classes. Though in general $J(\boldsymbol{\lambda} ; \mathbf{C})$ is not concave in $\boldsymbol{\lambda}$, in most relevant cases the first-order conditions (3) are still sufficient as it is shown next.

\section{Characterization of the Optimal Static Prices}

We begin by studying two particular cases. Namely, when $\sum_{k=1}^{K} N_{k} \leq N$ and when $N_{k} \geq N, \forall k \in$ $\mathcal{K}$. Following Ross [15], we refer to these cases as complete-partitioning and complete-sharing and we study them in $\S 3.1$ and $\S 3.2$ respectively. In $\S 3.3$ we analyze the general case when $\sum_{k=1}^{K} N_{k}>N$ and $N_{k}<N$ for at least one class $k$.

\subsection{Single-class and complete-partitioning case.}

We start the second-order analysis with a single customer class. For that case, the system reduces to an $M / G / N / N$ queue, also known as Erlang's loss system. We first give a useful Lemma that is well-known in the literature (see for instance $[2,7]$ and the references therein). However, we provide a short proof that is interesting per se since Equation (4) here below shows that, in an $M / G / N / N$ system, the (discrete) concavity of the nonblocking probability with respect to $N$ follows from the (continuous) convexity of its reciprocal with respect to the offered load $y$, and vice-versa. We then use this result to show quasiconcavity of the revenue function.

Lemma 1 In an $M / G / N / N$ system, the reciprocal of the the nonblocking probability is convex with respect to the offered load.

Proof: Consider the reciprocal of the nonblocking probability in an $M / G / N / N$ system, $\bar{B}(y ; N)^{-1}=$ $G(y ; N) / G(y ; N-1)$, expressed as a function of the offered load $y$. For $N=1$ the reciprocal is linear in $y$ and the result is trivial. Now assume $N>1$. By differentiating the reciprocal twice we have that the second derivative is non-negative if and only if the following inequality holds:

$$
\left[(G(y ; N-1) G(y ; N-2)+G(y ; N) G(y ; N-3)) G(y ; N-1)^{2}-2 \cdot G(y ; N) G(y ; N-1) G(y ; N-2)^{2}\right] \leqslant 0 .
$$

Dividing by $G(y ; N) G(y ; N-1)^{2} G(y ; N-2)$ and rearranging terms we obtain:

$$
\frac{\partial^{2} \bar{B}(y ; N)^{-1}}{\partial y^{2}} \geqslant 0 \Leftrightarrow \bar{B}(y ; N)+\bar{B}(y ; N-2) \leqslant 2 \bar{B}(y ; N-1) .
$$


Then, the result follows from the (discrete) concavity of the nonblocking probability with respect to $N$ (see Wolff and Wang [20]).

Proposition 1 For the single-class case: (i) If $p(\lambda)$ is (strictly) concave, then $J(\lambda ; N)$ is (strictly) concave in $\lambda$; and (ii) If $R(\lambda)$ is (strictly) concave, then $J(\lambda ; N)$ is (strictly) quasiconcave in $\lambda$.

Proof: For a single-class, $J(\lambda)=p(\lambda) \cdot E[X(\lambda)]=R(\lambda) \cdot \bar{B}(\lambda) / \mu$. For ease of notation we omit $N$. Note that $E[X(\lambda)]$ is non-decreasing and concave in $\lambda$, see Proposition 1 in Ross and Yao [17] and Harel [5]. If $p(\lambda)$ is (strictly) concave, then $J(\lambda)$ is (strictly) concave since it is the product of a (strictly) concave and decreasing function and a concave but non-decreasing function. For the second case, $J(\lambda)$ can be written as the ratio of $R(\lambda) / \mu$, that is non-negative (strictly) concave, and $\bar{B}(\lambda)^{-1}$, that is positive convex from Lemma 1. Hence, $J(\lambda)$ is (strictly) quasiconcave (see Avriel et al. [1]).

Proposition 1 holds for the linear demand case as well as for $p(\lambda)=a \cdot \lambda^{-b}$ with $a>0$ and $0<b<1$, or when $\lambda(p)=a \cdot \exp (-b p)$ with $a, b>0$. In all these cases, $J(\lambda ; N)$ is differentiable, and therefore it is pseudoconcave, so the first-order conditions are sufficient (see section 3.6 of [1]). When strict quasiconcavity holds, problem $(P)$ is guaranteed to have a unique optimal solution. This result extends to multiple classes under complete-partitioning since each class can be optimized separately.

\subsection{Complete-sharing case.}

In the complete-sharing case the blocking probability is the same for all classes. In fact, the system is equivalent to an $M / G / N / N$ queue with offered load equal to $\sum_{k=1}^{K} \lambda_{k} / \mu_{k}$. We use this fact to prove that the revenue function is quasiconcave:

Proposition 2 Under complete-sharing, if $R_{k}\left(\lambda_{k}\right)$ is (strictly) concave, $\forall k \in \mathcal{K}$, then $J(\boldsymbol{\lambda} ; \mathbf{C})$ is (strictly) quasiconcave in $\boldsymbol{\lambda}$.

Proof: In this case $J(\boldsymbol{\lambda} ; N)=\left(\sum_{k=1}^{K} \frac{R_{k}\left(\lambda_{k}\right)}{\mu_{k}}\right) /(G(\boldsymbol{\lambda} ; N) / G(\boldsymbol{\lambda} ; N-1))$. From Lemma 1 , the inverse of the nonblocking probability is convex in $y=\sum_{k=1}^{K} \lambda_{k} / \mu_{k}$, and therefore it is also convex in $\boldsymbol{\lambda}$. Then, $J(\boldsymbol{\lambda} ; N)$ is quasiconcave in $\boldsymbol{\lambda}$ as it is the ratio of two functions, one non-negative concave and the other positive convex.

Under complete-sharing the first order conditions (3) dictate that, at the optimum, all active classes must yield the same marginal revenue, i.e., $R_{k}^{\prime}\left(\lambda_{k}^{*}\right)=\beta^{*}>0, \forall k \in \mathcal{K}$ such that $\lambda_{k}^{*}>0$. Using this observation, problem $(P)$ can be greatly simplified. Indeed, let $\phi_{k}=R_{k}^{\prime}(0), \forall k \in \mathcal{K}$, and renumber the classes such that $\phi_{1} \geq \phi_{2} \geq \cdots \geq \phi_{K} \geq \phi_{K+1} \equiv 0$. We introduce the following parametrization of the arrival rates: $\lambda_{k}^{P}(\beta)=R_{k}^{\prime-1}(\beta) 1_{\left[\beta<\phi_{k}\right]}$, where $1_{A}$ is the indicator function. If $R_{k}\left(\lambda_{k}\right)$ is not strictly concave, then the inverse must be generalized in the usual way, i.e., $R_{k}^{\prime-1}(\beta)=\inf \left\{\lambda \in\left[0, \lambda_{k}^{\max }\right] \mid R_{k}(\lambda)=\beta\right\}$. 
It can be shown that solving problem $(P)$ is equivalent to solving the single-dimension fixedpoint equation $\beta=\Delta J\left(\boldsymbol{\lambda}^{P}(\beta) ; N\right) \equiv J\left(\boldsymbol{\lambda}^{P}(\beta) ; N\right)-J\left(\boldsymbol{\lambda}^{P}(\beta) ; N-1\right)$, which has a unique interior solution in the interval $\left[0, \phi_{1}\right]$. The parametric revenue function $J\left(\boldsymbol{\lambda}^{P}(\beta)\right)$ is continuous on $\left[0, \phi_{1}\right]$ but in general is not differentiable at the break points $\phi_{k}$ where an additional class becomes active. However, the left- and right-hand derivatives always exist for any interior point. Moreover, it can be shown that $J\left(\boldsymbol{\lambda}^{P}(\beta)\right)$ is quasiconcave in $\beta$. As mentioned in $\S 1$, Ziya et al. [21] suggest a different parametrization for the single-link case. Though it also reduces the formulation to one dimension, which they exploit analytically, there is not much gain computationally since their reduction still requires solving a multi-dimensional knapsack problem. In that respect, our parametrization should be more convenient for applications as it only requires solving a one-dimensional fixed-point equation.

The top graph in Figure 2 shows the shape of $J\left(\boldsymbol{\lambda}^{P}(\beta)\right)$ for different values of $N$ and linear demands such that $\phi_{1}=30$ and $\phi_{2}=8$ (the latter is marked with a solid vertical line and corresponds to a non-differentiable point). In the bottom graph of Figure 2 we plot $\Delta J\left(\boldsymbol{\lambda}^{P}(\beta)\right)$. The optimal value of $J\left(\boldsymbol{\lambda}^{P}(\beta)\right)$ is obtained when $\Delta J\left(\boldsymbol{\lambda}^{P}(\beta)\right)$ intersects the $45^{\circ}$ line. If $\beta^{*}$ is the unique fixed-point, then we know that $\beta^{*}<\phi_{1}$, and therefore class 1 is always active, i.e. its optimal arrival rate is nonzero. If $\beta^{*}<\phi_{2}$, then class 2 is active, and so on. In general, all classes "to the right" of $\beta^{*}$ will be active. For example, in Figure 2, class 2 has a zero arrival rate when $N=10$ because $\beta^{*}>8$, but it becomes active when $N=15$ since then $\beta^{*}<8$.

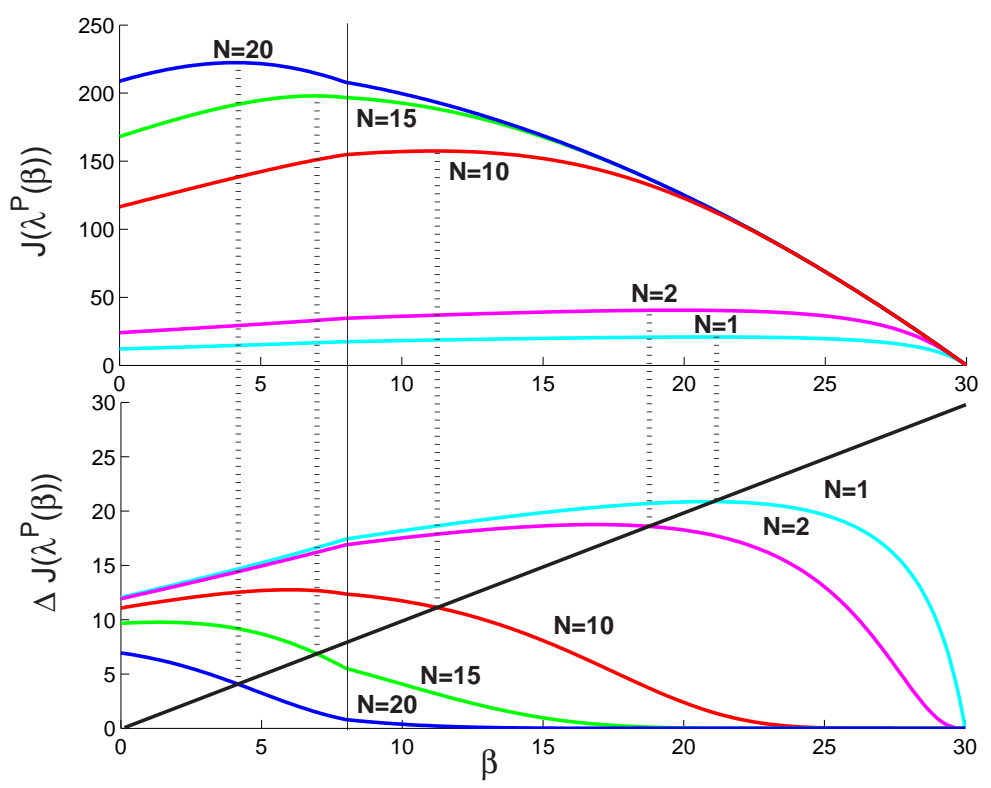

Figure 2: The parametric functions $J\left(\boldsymbol{\lambda}^{P}(\beta)\right)$ (top) and $\Delta J\left(\boldsymbol{\lambda}^{P}(\beta)\right)$ (bottom). The solid vertical line represents $\phi_{2}=8$. For easier representation, the vertical axis is not to scale.

The parametrization in terms of the marginal revenue $\beta$ allows us to show next the monotonicity of the optimal static prices $p_{k}^{*}(N)$ with respect to $N$. Once more, this is a direct consequence of the concavity of the nonblocking probabilities and serves as an alternative to the proof shown in Ziya et al. [21]. 
Proposition 3 Under complete-sharing, $p_{k}^{*}(N+1) \leq p_{k}^{*}(N), \forall k \in \mathcal{K}$, for any positive integer $N$.

Proof: Let $\beta_{a}$ and $\beta_{b}$ be the optimal solution to the fixed-point equation for $N$ and $N+1$ respectively. It suffices to show that $\beta_{a} \geq \beta_{b}$. The concavity of the nonblocking probabilities implies that:

$$
\Delta J\left(\boldsymbol{\lambda}^{P}(\beta) ; N\right) \geq \Delta J\left(\boldsymbol{\lambda}^{P}(\beta) ; N+1\right) \quad \forall \beta \in\left[0, \beta_{1}\right]
$$

In other words, $\Delta J\left(\boldsymbol{\lambda}^{P}(\beta) ; N\right)$ "lies above" $\Delta J\left(\boldsymbol{\lambda}^{P}(\beta) ; N+1\right)$. This remark provides an intuitive explanation of why $\beta_{a} \geq \beta_{b}$ must hold (see for example Figure 2). However, we continue with a formal proof in order to show that the concavity of the nonblocking probability has even stronger consequences. In fact, suppose that $\beta_{a}<\beta_{b}$. For $N>1$, the right-hand derivative of $\Delta J\left(\boldsymbol{\lambda}^{P}(\beta) ; N\right)$ at $\beta_{a}$ is given by

$$
\frac{\partial \Delta J\left(\boldsymbol{\lambda}^{P}\left(\beta_{a}^{+}\right) ; N\right)}{\partial \beta}=-\bar{B}\left(\boldsymbol{\lambda}^{P}\left(\beta_{a}\right)\right) \cdot\left(\Delta J\left(\boldsymbol{\lambda}^{P}\left(\beta_{a}\right) ; N\right)-\Delta J\left(\boldsymbol{\lambda}^{P}\left(\beta_{a}\right) ; N-1\right)\right) \cdot\left[\sum_{k=1}^{K} \frac{1}{\mu_{k}} \frac{\partial \lambda_{k}^{P}\left(\beta_{a}^{+}\right)}{\partial \beta}\right] .
$$

From Equation (5) we have that the right-hand derivative (6) is non-negative for $N>1$ (for $N=1$, $\Delta J\left(\boldsymbol{\lambda}^{P}(\beta) ; 1\right)=J\left(\boldsymbol{\lambda}^{P}(\beta) ; 1\right)$ so the derivative is zero at $\left.\beta_{a}\right)$. Then, there must exist $\widehat{\beta}>\beta_{a}$ such that $\Delta J\left(\boldsymbol{\lambda}^{P}(\widehat{\beta}) ; N\right)<\widehat{\beta}$. Let $q(\beta) \equiv \Delta J\left(\boldsymbol{\lambda}^{P}(\beta) ; N\right)-\beta$, which is a continuous function of $\beta$. On the one hand we have $q(\widehat{\beta})<0$, and on the other hand, from $\beta_{b}=\Delta J\left(\boldsymbol{\lambda}^{P}\left(\beta_{b}\right) ; N+1\right)$ and (5), we have that $q\left(\beta_{b}\right) \geq 0$. Then there must exist $\xi \in\left[\min \left(\widehat{\beta}, \beta_{b}\right), \max \left(\widehat{\beta}, \beta_{b}\right)\right]$ such that $q(\xi)=0$. This implies $\xi=\beta_{a}$ (from the uniqueness of $\beta_{a}$ ), which is a contradiction since $\xi \geq \min \left(\widehat{\beta}, \beta_{b}\right)>\beta_{a}$. Consequently, it must be that $\beta_{a} \geq \beta_{b}$, and the proof is complete.

\subsection{General case.}

For the general case we would like to identify when the following two conditions hold: $(i)$ the upper-level sets of the revenue function are connected, and $(i i)$ the optimal solution of $(P)$ is unique. Condition $(i)$ is necessary (and sufficient under proper assumptions) for a greedy search method to converge to a global maximum. Condition (ii) is a desirable property that allows the construction of one-to-one mappings as we did in $\S 3.2$. A sufficient condition for $(i)$ and $(i i)$, which we denote $(S)$, is the following: considering all $K$ classes or less, the hessian of the revenue function at any interior solution of the first-order conditions is definite negative. Condition $(S)$ requires any solution to the first-order conditions (3) to be a strict local maximum, which implies that a critical point is a unique global maximizer. Moreover, it can be shown that condition $(S)$ implies that the upper-level sets are connected, see Proposition 3 in Martínez-de-Albéniz and Simchi-Levi [10] and the discussion thereafter. Note that in general condition $(S)$ is weaker than quasiconcavity. In fact, there exist functions that do not have convex upper-level sets, i.e., are not quasiconcave, but do satisfy condition $(S)$, see for instance Figure 2 in [10].

Let $\boldsymbol{\lambda}^{*}>0$ be a feasible point for $(P)$ that satisfies the first-order conditions (3). The hessian 
of the revenue function at $\boldsymbol{\lambda}^{*}$ can then be decomposed as:

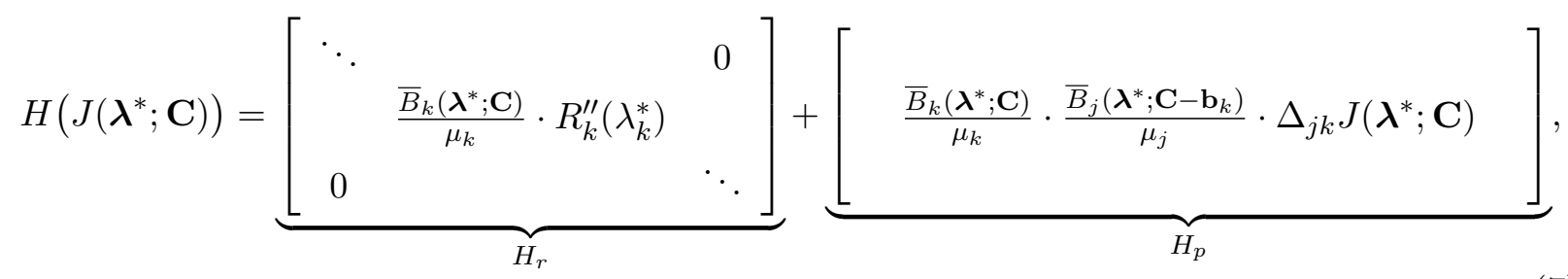

where $\Delta_{j k} J(\boldsymbol{\lambda} ; \mathbf{C}) \equiv J(\boldsymbol{\lambda} ; \mathbf{C})-J\left(\boldsymbol{\lambda} ; \mathbf{C}-\mathbf{b}_{j}\right)-J\left(\boldsymbol{\lambda} ; \mathbf{C}-\mathbf{b}_{k}\right)+J\left(\boldsymbol{\lambda} ; \mathbf{C}-\mathbf{b}_{j}-\mathbf{b}_{k}\right)$ is the double backward difference operator. Then, for condition $(S)$ to hold, we must verify that $(7)$ is definite negative, considering all $K$ classes or less. When all $R_{k}$ are strictly concave, clearly the diagonal matrix $H_{r}$ is definite negative. The contribution of $H_{p}$ depends on the capacity vector $\mathbf{C}$. For instance, as expected, under complete-partitioning the matrix $H_{p}$ becomes a diagonal matrix with non-positive entries. However, the main result in this subsection is negative: for a general tree network, condition $(S)$ does not hold. In fact, consider a system with two classes and linear demands $\lambda_{k}\left(p_{k}\right)=\alpha_{k}-p_{k} \gamma_{k}$. The capacities of the common link and the outbound links are respectively $N=2, N_{1}=2$, and $N_{2}=1$. Assuming, $\gamma_{1}=1 / 9, \alpha_{1} / \gamma_{1}=30, \gamma_{2}=1 / 7, \alpha_{2} / \gamma_{2}=0.02$, and $\mu_{1}=\mu_{2}=1$, we can see in Figure 3 that the revenue function has an inflexion point and the upper-level sets are not connected.

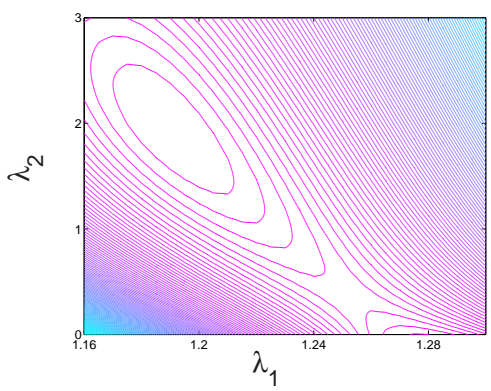

Figure 3: Disconnected level sets for the general case.

The reason for condition $(S)$ not to hold in the previous example is that $N_{2}=1$ implies $\bar{B}_{2}\left(\boldsymbol{\lambda}^{*} ; \mathbf{C}-\mathbf{b}_{2}\right)=0$ so the lower right entry of $H_{p}$ is zero, meaning that $H_{p}$ can never be semidefinite negative. Then, we can chose the problem parameters so that $H\left(W\left(\boldsymbol{\lambda}^{*} ; \mathbf{C}\right)\right)$ becomes indefinite. From the previous observation, it seems that having one of the outbound links with unit capacity is problematic. Note also that the case $N=1$ was always treated differently in the proofs in $§ 3.2$. The immediate question is: does condition $(S)$ hold if $N_{k}>1, \forall k \in \mathcal{K}$ ? We address this question numerically in $\S 5$ and we conjecture that the answer is affirmative when demands are linear.

To solve the static pricing problem for a general tree network, we suggest a parametrization of the first-order conditions (3) that lead to a fixed-point equation analogous to the one derived for the complete-sharing case. Formally, let $\beta_{k}$ be a non-negative vector where the $k$-th component represents the opportunity cost of admitting a class- $k$ call. Then we have

$$
\lambda_{k}^{P}(\boldsymbol{\beta})=R_{k}^{\prime-1}\left(\beta_{k}\right) 1_{\left[\beta_{k}<\phi_{k}\right]}, \quad \forall k \in \mathcal{K},
$$




$$
\beta_{k}=J\left(\boldsymbol{\lambda}^{P}(\boldsymbol{\beta}) ; \mathbf{C}\right)-J\left(\boldsymbol{\lambda}^{P}(\boldsymbol{\beta}) ; \mathbf{C}-\mathbf{b}_{k}\right), \quad \forall k \text { such that } \beta_{k}<\phi_{k},
$$

where $\phi_{k}$ is defined as before. Equation (9) provides a critical point and a global maximum if condition $(S)$ holds. Though it is a $K$-dimensional fixed-point equation, it can still be solved by repeated substitution. The main challenge could eventually be the computation of the non-blocking probabilities to evaluate the revenue function. However, for practical applications we suggest using the reduced load approximation (see [15]), which in the case of a tree network amounts to solving another single-dimensional fixed-point equation given by

$$
L=\bar{E}\left(\sum_{k \in \mathcal{K}} y_{k} \bar{E}\left(y_{k} L ; N_{k}\right) ; N\right),
$$

where $\bar{E}(\rho ; N)$ is the non-blocking probability in an Erlang system with offered load $\rho$ and capacity $N$. Again, Equation (10) can be solved efficiently through repeated substitution, and then the nonblocking probability for class- $k$ is computed approximately as $\bar{B}_{k} \approx L \bar{E}\left(y_{k} L ; N_{k}\right)$.

\section{Asymptotically Optimal Prices}

We now consider a relaxation of the original static pricing problem $(P)$ to obtain an upper bound on the optimal revenue. This is the same upper bound as in $[9,12,13]$, but our derivation is specific to the static pricing case and does not require the functions $R_{k}\left(\lambda_{k}\right)$ to be concave. Instead, we use the fact that $p_{k}\left(\lambda_{k}\right)$ is decreasing, $\forall k \in \mathcal{K}$.

Suppose that the service provider is allowed to admit an unlimited number of calls, but in steady-state the occupancy of the links must not exceed the former nominal capacities on average (where the average is with respect to the steady-state probabilities). In this relaxed problem, that we call $\left(P_{u b}\right)$, the links correspond to independent $M / G / \infty$ queues. Then, the average number of customers is equal to the offered load, and we have that problem $\left(P_{u b}\right)$ can be formulated as the following nonlinear program:

$$
\begin{array}{lll}
\left(P_{u b}\right) \quad \max & \sum_{k=1}^{K} R_{k}\left(\lambda_{k}\right) / \mu_{k} \\
& \text { s.t. } & \sum_{k=1}^{K} \lambda_{k} / \mu_{k} \leq N \\
& & \lambda_{k} / \mu_{k} \leq N_{k}, \forall k \in \mathcal{K} .
\end{array}
$$

We now show that the relaxed problem $\left(P_{u b}\right)$ indeed provides an upper bound.

Proposition 4 Assume $p_{k}\left(\lambda_{k}\right)$ is decreasing, $\forall k \in \mathcal{K}$, and let $J^{*}$ and $J^{u b}$ be the optimal value of $(P)$ and $\left(P_{u b}\right)$ respectively. Then, $J^{*} \leq J^{u b}$.

Proof: Let $\boldsymbol{\lambda}^{*}$ be the optimal solution to the static pricing problem $(P)$. Since at any point in time in the original (non-relaxed) system we have that $X_{k}\left(\boldsymbol{\lambda}^{*} ; \mathbf{C}\right) \leq N_{k}, \forall k \in \mathcal{K}$, and $\sum_{k \in \mathcal{K}} X_{k}\left(\boldsymbol{\lambda}^{*} ; \mathbf{C}\right) \leq$ $N$, then the constraints must also be satisfied on average. That is, $E_{k}^{*} \equiv \mathbb{E}\left[X_{k}\left(\boldsymbol{\lambda}^{*} ; \mathbf{C}\right)\right] \leq N_{k}, \forall k \in$ $\mathcal{K}$, and $\sum_{k \in \mathcal{K}} E_{k}^{*} \leq N$. Therefore, the solution $E_{k}^{*} \mu_{k}, k \in \mathcal{K}$, is feasible for problem $\left(P_{u b}\right)$, so we can write

$$
J^{u b} \geq \sum_{k \in \mathcal{K}} p_{k}\left(E_{k}^{*} \mu_{k}\right) E_{k}^{*} \geq \sum_{k \in \mathcal{K}} p_{k}\left(\frac{E_{k}^{*} \mu_{k}}{\bar{B}_{k}\left(\boldsymbol{\lambda}^{*} ; \mathbf{C}\right)}\right) E_{k}^{*}=J^{*}
$$


where the second inequality is because $p_{k}\left(\lambda_{k}\right)$ is decreasing, $\forall k \in \mathcal{K}$, and the last equality follows from Little's Law and the optimality of $\boldsymbol{\lambda}^{*}$.

Besides the upper bound, problem $\left(J^{u b}\right)$ provides a static solution $\boldsymbol{\lambda}^{u b}$ (or $\mathbf{p}^{u b}$ ) for the original problem $(P)$. If the capacity and the arrival rates $\boldsymbol{\lambda}^{u b}$ are increased by the same factor, then it can be shown that as the factor tends to infinity, the blocking probabilities tend to zero (see $[9])$. Hence, the objective value of the scaled system evaluated in $\mathbf{p}^{u b}$ asymptotically reaches the upper bound $J^{u b}$, and for that reason we will refer to $\mathbf{p}^{u b}$ as the asymptotically optimal prices. Given this asymptotic property, and since problem $\left(J^{u b}\right)$ is fairly easy to solve, the prices $\mathbf{p}^{u b}$ are a natural candidate to use as an approximate solution for problem $(P)$. In the numerical section we study the performance of $\mathbf{p}^{u b}$ versus the optimal static prices and those computed heuristically by approximating the non-blocking probabilities. Finally, note that when the instantaneous reward functions $R_{k}\left(\lambda_{k}\right)$ are concave, then $J^{u b}$ is also a valid upper bound for any dynamic pricing policy (see $[13])$.

We conclude this section providing bounds on the performance of the asymptotically optimal static prices. For that, we first need the lemma below which shows that $\bar{E}(N ; N)$ is increasing in $N$. Note that this result is non-trivial since $\bar{E}(\rho ; N)$ is decreasing in $\rho$ for a given $N$ so the fact that $\bar{E}(N ; N)$ increases with $N$ is not straightforward.

Lemma 2 Let $N$ be a positive integer, then $\bar{E}(N ; N)$ is strictly monotone increasing in $N$.

Proof: The non-blocking probability in an Erlang loss system is given by $\bar{E}(\rho ; N)=1-\frac{\rho^{N} / N !}{\sum_{i=0}^{N} \rho^{i} / i !}$. We would like to show that $\bar{E}(N ; N)<\bar{E}(N+1 ; N+1)$ for any $N$ non-negative integer, which is equivalent to showing

$$
\sum_{i=0}^{N+1} \frac{(N+1)^{i}}{i !}>\left(\sum_{i=0}^{N} \frac{N^{i}}{i !}\right)\left(1+\frac{1}{N}\right)^{N}, \quad \forall N \in \mathbb{N} \cup\{0\} .
$$

We show this directly:

$$
\begin{aligned}
\sum_{i=0}^{N+1} \frac{(N+1)^{i}}{i !} & =\frac{(N+1)^{N}}{N !}+\sum_{i=0}^{N} \frac{(N+1)^{i}}{i !} \\
& =\frac{(N+1)^{N}}{N !}+\sum_{i=0}^{N} \sum_{j=0}^{i} \frac{N^{j}}{j !(i-j) !}
\end{aligned}
$$

(By the binomial theorem)

$$
=\frac{(N+1)^{N}}{N !}+\sum_{j=0}^{N} \frac{N^{j}}{j !}\left(\sum_{k=0}^{N-j} \frac{1}{k !}\right)
$$

(By interchanging the summation order and then defining $i-j$ as $k$ )

$$
=\frac{(N+1)^{N}}{N !}+\sum_{j=0}^{N-1} \frac{N^{j}}{j !}\left(\sum_{k=0}^{N} \frac{1}{k !}\right)+\frac{N^{N}}{N !}\left(1-\sum_{j=1}^{N-1} \frac{N !}{N^{N}} \frac{N^{j}}{j !}\left(\sum_{k=N-j+1}^{N} \frac{1}{k !}\right)\right)
$$

(By rearranging terms) 


$$
\geq \frac{(N+1)^{N}}{N !}+\sum_{j=0}^{N-1} \frac{N^{j}}{j !}\left(\sum_{k=0}^{N} \frac{1}{k !}\right)+\frac{N^{N}}{N !}\left(1-\sum_{j=1}^{N-1}\left(\sum_{k=N-j+1}^{N} \frac{1}{k !}\right)\right)
$$

(Because $N$ is the mode of a Poisson random variable with parameter $N$

$$
\begin{aligned}
& \text { so } \left.N^{j} / j ! \leq N^{N} / N !, \forall j \leq N\right) \\
= & \frac{(N+1)^{N}}{N !}+\sum_{j=0}^{N-1} \frac{N^{j}}{j !}\left(\sum_{k=0}^{N} \frac{1}{k !}\right)+\frac{N^{N}}{N !}\left(1-\sum_{j=1}^{N-1}\left(\sum_{\ell=1}^{j} \frac{1}{(N-\ell+1) !}\right)\right)
\end{aligned}
$$

(By defining $N-k+1$ as $\ell$ )

$$
=\frac{(N+1)^{N}}{N !}+\sum_{j=0}^{N-1} \frac{N^{j}}{j !}\left(\sum_{k=0}^{N} \frac{1}{k !}\right)+\frac{N^{N}}{N !}\left(1-\sum_{\ell=1}^{N-1}(N-\ell) \frac{1}{(N-\ell+1) !}\right)
$$

(By interchanging the order of the last two summations)

$$
=\frac{(N+1)^{N}}{N !}+\sum_{j=0}^{N-1} \frac{N^{j}}{j !}\left(\sum_{k=0}^{N} \frac{1}{k !}\right)+\frac{N^{N}}{N !}\left(1-\sum_{\ell=1}^{N-1}\left(\frac{1}{(N-\ell) !}-\frac{1}{(N-\ell+1) !}\right)\right)
$$

(By using some algebra to rewrite the term in the last summation)

$$
=\frac{(N+1)^{N}}{N !}+\sum_{j=0}^{N-1} \frac{N^{j}}{j !}\left(\sum_{k=0}^{N} \frac{1}{k !}\right)+\frac{N^{N}}{N !}\left(\frac{1}{N !}\right)
$$

(By telescoping the last sum)

$$
\geq \frac{(N+1)^{N}}{N !}+\sum_{j=0}^{N-1} \frac{N^{j}}{j !}\left(1+\frac{1}{N}\right)^{N}+\frac{N^{N}}{(N !)^{2}}
$$

(By theorem 3.31 in Rudin [18])

$$
\begin{aligned}
& =\sum_{j=0}^{N} \frac{N^{j}}{j !}\left(1+\frac{1}{N}\right)^{N}+\frac{N^{N}}{(N !)^{2}} \\
& >\sum_{j=0}^{N} \frac{N^{j}}{j !}\left(1+\frac{1}{N}\right)^{N},
\end{aligned}
$$

and the proof is complete.

Finally, the following lemma provides the bounds. Let $\lambda^{u b}$ be the optimal solution to (11) and let $y_{k}^{u b}=\lambda_{k}^{u b} / \mu_{k}, \forall k \in \mathcal{K}$.

Lemma 3 Let $M=\min _{k \in \mathcal{K}} N_{k}$ and $\rho^{u b}=\sum_{k \in \mathcal{K}} y_{k}^{u b}$, then:

$$
1 \geq \frac{J\left(\boldsymbol{\lambda}^{u b} ; \mathbf{C}\right)}{J^{u b}} \geq \min _{k} \bar{B}_{k}\left(\boldsymbol{\lambda}^{u b} ; \mathbf{C}\right) \geq \min _{k \in \mathcal{K}} \bar{E}\left(\rho^{u b} ; N\right) \bar{E}\left(y_{k}^{u b} ; N_{k}\right) \geq \bar{E}(N ; N) \bar{E}(M ; M) .
$$

Proof: The first and second inequalities follow from the definition of $J^{u b}$ and $J\left(\boldsymbol{\lambda}^{u b} ; \mathbf{C}\right)$. The third inequality follows from the independent link bound for loss systems (see [15]). The last inequality uses Lemma 2 and the worst case scenario $\rho^{u b}=N$ and $y_{k}^{u b}=N_{k}, \forall k \in \mathcal{K}$. 


\section{Numerical Analysis}

In this section we provide some illustrative numerical examples with linear demands $\lambda_{k}\left(p_{k}\right)=$ $\alpha_{k}-p_{k} \gamma_{k}$. The goal is twofold: (i) analyze and discuss the relationship between problem parameters and the solution structure; and (ii) compare the performance of the asymptotically optimal, reduced load approximation, and optimal static prices.

We first consider a two-class complete-sharing system with $\alpha_{k}=10 \gamma_{k}, \gamma_{1}=100, \gamma_{2}=20$, and $\mu_{k}=k$. Since $\phi_{1}=\phi_{2}=10$, from $\S 3.2$ we know that both classes are "attractive" to the network service provider. Moreover, from the optimality conditions (3) it follows that the prices for both classes are equal. In Table 1 we compare the performance of the asymptotic and optimal static prices for increasing values of $N$. We observe that $p_{k}^{u b}>p_{k}^{*}$, which is intuitive (see the proof of Proposition 4), and we also confirm the monotonicity property from Proposition 3 . When the blocking level goes down, so does the suboptimality gap of the asymptotic prices. Since the relaxed problem $P_{u b}$ does not have blocking, it is clear that the asymptotically optimal prices $p_{k}^{u b}$ will perform well if they induce blocking probabilities that are close to zero. This fact is confirmed by the convergence rate proved in [13] which shows that the system must be scaled until there is no blocking for the asymptotic result to take place.

\begin{tabular}{c||ccc|cc|c|cc}
\hline$N$ & $J\left(\mathbf{p}^{u b} ; \mathbf{C}\right)$ & $J^{*}$ & $J^{u b}$ & $p_{k}^{u b}$ & $p_{k}^{*}$ & Blocking & $\frac{J^{u b}-J\left(\mathbf{p}^{u b} ; \mathbf{C}\right)}{J^{u b}}$ & $\frac{J^{u b}-J^{*}}{J^{u b}}$ \\
\hline \hline 5 & 35.59 & 46.91 & 49.77 & 9.95 & 9.67 & 0.86 & $28.49 \%$ & $5.75 \%$ \\
10 & 77.83 & 93.40 & 99.09 & 9.91 & 9.63 & 0.76 & $21.46 \%$ & $5.74 \%$ \\
20 & 165.16 & 185.14 & 196.36 & 9.82 & 9.55 & 0.61 & $15.89 \%$ & $5.71 \%$ \\
40 & 340.68 & 363.63 & 385.45 & 9.64 & 9.39 & 0.43 & $11.61 \%$ & $5.66 \%$ \\
60 & 512.66 & 535.49 & 567.27 & 9.45 & 9.22 & 0.32 & $9.63 \%$ & $5.60 \%$ \\
90 & 760.61 & 780.84 & 826.36 & 9.18 & 8.98 & 0.23 & $7.96 \%$ & $5.51 \%$ \\
\hline
\end{tabular}

Table 1: Asymptotically optimal $\mathbf{p}^{u b}$ and optimal static $\mathbf{p}^{*}$ prices under complete-sharing.

The next set of numerical experiments studies the performance of a two-class general tree network. The demand functions are the same as before and we excluded cases with $N_{1}=1$ or $N_{2}=1$ since, as discussed above, the revenue level sets might not be connected. In particular, we considered instances with $N, N_{1} \in\{10, \ldots, 20\}$ and $N_{2}=10$. We used third-party algorithms [19] to verify condition $(S)$ (c.f. $§ 3.3$ ). The conclusion was that condition $(S)$ holds in all the cases considered, for any given set of parameters. To compute the non-blocking probabilities, we used the normalization constant algorithm in Pinsky and Conway [14]. We chose this general algorithm instead of a specialized routine for a tree network (such as Ross and Tsang [16]) because it can easily handle numerical overflows which occur frequently even for mid-size instances.

In Figure 4 we perform a sensitivity analysis for the optimal static prices with respect to problem parameters. In the left graph we keep everything fixed except for the demand intercept $\alpha_{1}$. By increasing $\alpha_{1}$, the demand for that class becomes more inelastic. As expected, $p_{1}^{*}$ increases since the service provider can charge more and make a higher profit. The price $p_{2}^{*}$ is also increasing (though less pronounced), which reflects the fact that the capacity opportunity cost is higher. The middle graph of Figure 4 shows the sensitivity with respect to $N_{1}$. Interestingly, $p_{1}^{*}$ is not monotone as the system transitions through the three possible structures we have covered: complete-partitioning 

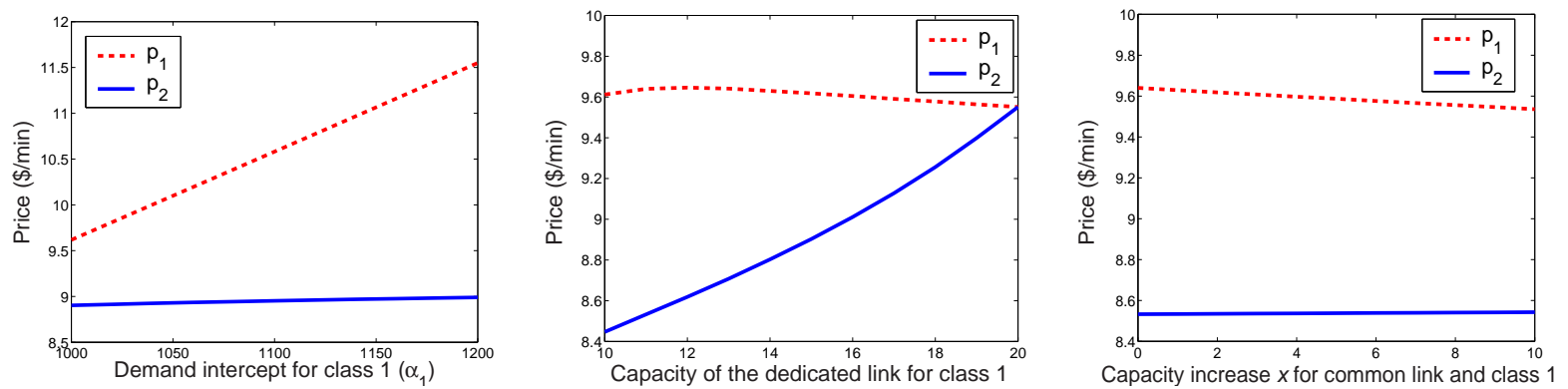

Figure 4: Sensitivity of the optimal static prices with respect to: demand intercept $\alpha_{1}$ (left graph, $N=20, N_{1}=15$ ), outbound capacity $N_{1}$ (middle graph, $N=20$ ), and capacity increase $x$ (right graph, $\left.N=20+x, N_{1}=11+x\right)$, in a two-class tree network with $N_{2}=10$.

$\left(N_{1}=10\right)$, general case $\left(N_{1}=11, \ldots, 19\right)$, and complete-sharing $\left(N_{1}=20\right) .{ }^{1}$ The monotonicity property is recovered if the capacity on both links used by class- 1 increases simultaneously. This is shown in the right graph of Figure 4, which would be the equivalent to Proposition 3 in the general case.

For the instances in the middle graph of Figure 4 we computed the steady-state revenue for the asymptotically optimal, reduced load approximation, optimal static prices. These values are reported in Table 2, first in absolute terms, and then as a percentage difference with respect to the upper bound $J^{u b}$. From Equations (8)-(10) it can be seen that the effort needed to compute the reduced load approximation prices is comparable to finding the asymptotically optimal ones. However, the former outperforms the latter, as it can be seen in Table 2. Indeed, the reduced load approximation prices yield a steady-state revenue that is only slightly worse than the optimal static, whereas the asymptotically optimal prices are consistently ten percentage point below. Note that the gap with respect to $J^{u b}$ could suggest that a dynamic pricing policy has room to do better, but that is not clear as in these cases we believe that the bound is just not tight enough.

\begin{tabular}{c|cccc|ccc}
\hline$N_{1}$ & $J\left(\mathbf{p}^{u b} ; \mathbf{C}\right)$ & $J\left(\mathbf{p}^{r} ; \mathbf{C}\right)$ & $J^{*}$ & $J^{u b}$ & $\frac{J^{u b}-J\left(\mathbf{p}^{u b} ; \mathbf{C}\right)}{J^{u b}}$ & $\frac{J^{u b}-J\left(\mathbf{p}^{r} ; \mathbf{C}\right)}{J^{u b}}$ & $\frac{J^{u b}-J^{*}}{J^{u b}}$ \\
\hline \hline 10 & 148.44 & 168.14 & 168.26 & 189.00 & $21.46 \%$ & $11.04 \%$ & $10.97 \%$ \\
12 & 155.89 & 174.13 & 174.44 & 192.16 & $18.87 \%$ & $9.38 \%$ & $9.22 \%$ \\
14 & 159.35 & 178.44 & 178.57 & 194.44 & $18.05 \%$ & $8.23 \%$ & $8.16 \%$ \\
16 & 161.22 & 181.66 & 181.75 & 195.84 & $17.68 \%$ & $7.24 \%$ & $7.19 \%$ \\
18 & 162.54 & 183.96 & 184.01 & 196.36 & $17.22 \%$ & $6.31 \%$ & $6.29 \%$ \\
20 & 165.16 & 185.07 & 185.14 & 196.36 & $15.89 \%$ & $5.75 \%$ & $5.72 \%$ \\
\hline
\end{tabular}

Table 2: Percentage difference with respect to $J^{u b}$ for the asymptotically optimal $\mathbf{p}^{u b}$, reduced load approximation $\mathbf{p}^{r}$, and optimal static $\mathbf{p}^{*}$ prices, in a two-class tree network $\left(N=20, N_{2}=10\right)$.

To further investigate the performance gap between the asymptotic and optimal static prices, we fix the common link capacity $N=100$ and we consider $K$ identical classes with $\alpha_{k}=5120, \gamma_{k}=$ $512, \mu_{k}=1$ and $N_{k}=M, \forall k \in \mathcal{K}$. Note that if all $\alpha_{k}$ are equal, or all $\gamma_{k}$ are equal, then condition $(S)$ can be easily verified analytically through direct calculation of the corresponding

\footnotetext{
${ }^{1}$ In this last case, $N_{2}<N$ but it is large enough to fall in the complete-sharing case. That is why the two prices are identical.
} 
determinants (despite the extremely lengthy expressions, determining the sign of the inequalities is straightforward). This provides additional support to our belief that condition $(S)$ is satisfied in general when $N_{k}>1, \forall k \in \mathcal{K}$, and demands are linear.
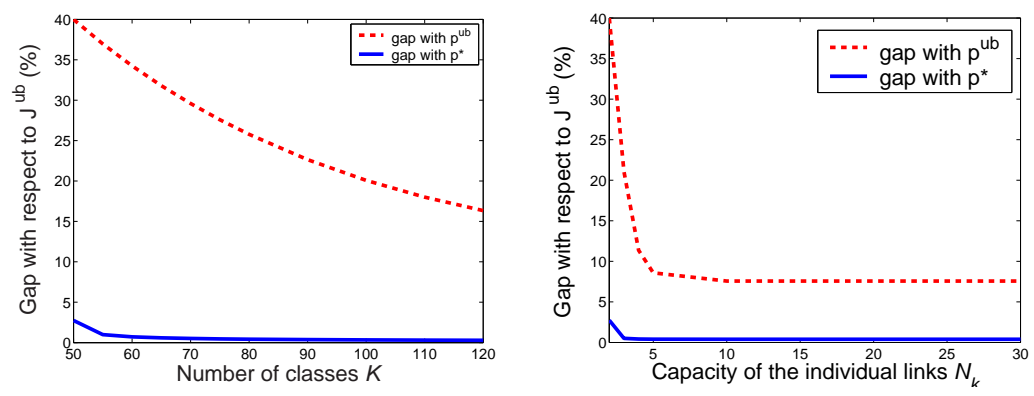

Figure 5: Percentage difference with respect to $J^{u b}$ for symmetric classes as a function of the number of classes $K$ (left graph, $N_{k}=2$ ) and the outbound capacity $N_{k}$ (right graph, $K=50$ ).

In Figure 5 we plot the percentage difference of the steady-state revenue with respect to the upper bound $J^{u b}$ for the asymptotically optimal and optimal static prices as a function of $K$ and $N_{k}$. The percentage difference for the asymptotically optimal prices is significant whereas for the optimal static prices it quickly becomes minimal. This implies that even a dynamic pricing policy has little room for improvement over the optimal static prices. For this symmetric example, the next-to-last bound in (13) yields $\frac{J^{u b}-J\left(\boldsymbol{\lambda}^{u b} ; \mathbf{C}\right)}{J^{u b}}=B_{k}\left(\boldsymbol{\lambda}^{u b} ; \mathbf{C}\right) \leq 1-\bar{E}(100 ; 100) \bar{E}\left(\frac{N}{K} ; N_{k}\right)$. The latter tends to $1-\bar{E}(100 ; 100)=7.57 \%$ as either $K$ or $N_{k}$ goes to infinity. In both graphs in Figure 5 , the curve with $p^{u b}$ tends to the bound from above. The same observation can be made for any capacity level $N$. Moreover, in all these cases, the reduced load approximation yields the optimal static prices, which confirms even further that this approach seems preferable for systems outside the asymptotic regime of $\left(P^{u b}\right)$. As for when the asymptotically optimal prices can be good enough, the last inequality in (13) provides an indicator. This bound does not depend on the demand parameters and can be easily tabulated for a quick reference.

\section{Conclusions and Extensions}

In this paper we presented a loss model for the pricing problem in a tree network. The work was motivated by a company that sells phone cards. We restricted our study to static pricing policies and focused on finding the optimal policy within that subclass. The first-order optimality conditions have the same interpretation as many other revenue management problems: the optimal prices must balance marginal revenue with opportunity costs. In terms of second-order conditions, for two important cases, complete-partitioning and complete-sharing, it was shown that the revenue function is quasiconcave. This property does not extend to the general tree network case, in particular, when one of the outbound links has a single circuit (i.e., its capacity is equal to one). Numerical evidence indicates that the latter seems to be the only problematic case, at least when the demand functions are linear.

We then compared the performance of the optimal static prices with those obtained from solving an asymptotic approximation where call blocking becomes negligible. We show that outside the 
the asymptotic regime there can be a significant gain from using the optimal static prices. Since evaluating the normalization constant remains a computational challenge, our results suggest using prices obtained with the reduced load approximation for the blocking probabilities.

We conclude the paper discussing which of our results extend to more general settings:

- Demand substitution: We assumed that the demand (arrival rate) of class $k$ only depends on its own price. This dependence can be extended to the complete price vector to allow for demand substitution. Additional consistency assumptions, as those given in [3, 13], might be needed. Assuming that the demand function is invertible, the instantaneous rewards $R_{k}(\boldsymbol{\lambda})=\lambda_{k} p_{k}(\boldsymbol{\lambda})$ are now a function of the arrival rate vector. The first-order conditions (for $\left.\lambda_{k}^{*}>0\right)$ become:

$$
\sum_{j=1}^{K} \frac{\partial R_{j}\left(\boldsymbol{\lambda}^{*}\right)}{\partial \lambda_{k}} \cdot \frac{\bar{B}_{j}\left(\boldsymbol{\lambda}^{*}\right)}{\mu_{j}}=\frac{\bar{B}_{k}\left(\boldsymbol{\lambda}^{*}\right)}{\mu_{k}} \cdot\left[J\left(\boldsymbol{\lambda}^{*} ; \mathbf{C}\right)-J\left(\boldsymbol{\lambda}^{*} ; \mathbf{C}-\mathbf{b}_{k}\right)\right] .
$$

Note that each term is weighted by the corresponding nonblocking probability and holding time, but the interpretation of "marginal revenue equal to opportunity cost" remains valid. The results of $\S 3.1$ for the complete-partitioning case no longer hold since the objective function is not separable. If $R_{k}(\boldsymbol{\lambda})$ is jointly concave for all $k$, then the proof of Proposition 2 for the complete-sharing case is the same. In this case, the nonblocking probabilities in (14) cancel out. If the equations

$$
\sum_{j=1}^{K} \frac{\partial R_{j}(\boldsymbol{\lambda})}{\partial \lambda_{k}} \cdot \frac{\mu_{k}}{\mu_{j}}=\beta, \quad \forall k \in \mathcal{K},
$$

can be solved uniquely for $\boldsymbol{\lambda}$ as a function of $\beta$, then problem $(P)$ can be reduced to a single dimension as we did in $§ 3.2$. For a general tree network, the fixed-point formulation (8)-(9) breaks down, though of course the reduced load approximation (10) can still be used. Finally, in $\S 4$, Proposition 4 and Lemma 3 would now require concavity of the instantaneous reward rates.

- Heterogeneous resource requirements: When customer classes have different bandwidth requirement, the first-order conditions (3) hold with the vector $\mathbf{b}_{k}$ appropriately defined. The results for complete-partitioning also follow, but those for complete-sharing do not since the blocking probabilities are no longer equal. The rest of the analysis remains unchanged. In particular, Equation (7) remains valid, so having $\mathbf{C}-\mathbf{b}_{k}>0, \forall k \in \mathcal{K}$ is necessary for condition $(S)$ to hold.

- General product form loss network: When the loss network has a general structure, as long as the stationary probability distribution has a product form, then the first-order conditions (3) will hold and the discussions in $\S 3.3$ and $\S 4$ will follow. However, calculating the normalization constant for a general loss network is an NP-complete problem (see [15]), so computing static prices using an approximation of the blocking probabilities becomes even more relevant. 


\section{References}

[1] M. Avriel, W.E. Diewert, S. Schaible and I. Zang, Generalized Concavity, Plenum Publishing, New York (1988).

[2] E. Carrizosa, E. Conde and M. Muñoz-Márquez, Admission Policies in Loss Queueing Models with Heterogeneous Arrivals, Management Science, 44(3) (1998) 311-320.

[3] C.A. Courcoubetis and M.I. Reiman, Pricing in a Large Single Link Loss System, in: Teletraffic Engine in a Competitive World, eds. P. Key and D. Smith, Elsevier-Amsterdam (1999) 737-746.

[4] C.A. Courcoubetis and R.R. Weber, Pricing Communication Networks, John Wiley \& Sons, West Sussx, England (2003).

[5] A. Harel, Convexity Properties of the Erlang Loss Formula, Ops. Res., 38(3) (1990) 499-505.

[6] F.P. Kelly, Loss Networks, Annals of Applied Probability, 1(3) (1991) 319-378.

[7] J.R. Kenevan and A.K. von Mayrhauser, Convexity and Concavity Properties of Analytic Queueing Models for Computer Systems, in: Performance '84, ed. E. Gelenbe, Elsevier Science Publishers B.V., North-Holland, (1984), pp. 361-375.

[8] S. Lanning, W.A. Massey, B. Rider and Q. Wang, Optimal Pricing in Queueing Systems with Quality of Service Constraints, in: Teletraffic Engineering in a Competitive World, eds. P. Key and D. Smith, Elsevier-Amsterdam (1999) 747-757.

[9] X. Lin and N.B. Shroff, Simplification of Network Dynamics in Large Systems, IEEE/ACM Transactions on Networking, 13(4) (2005) 813-826.

[10] V. Martínez-de-Albéniz and D. Simchi-Levi, Mean-Variance Trade-offs in Supply Contracts, Naval Research Logistics, 53(7) (2006) 603-616.

[11] H. Mendelson, Pricing Computer Services: Queueing Effects, Communications of the ACM, 28(3) (1985) 312-321.

[12] I.Ch. Paschalidis and J.N. Tsitsiklis, Congestion-Dependent Pricing of Network Services, IEEE/ACM Transactions on Networking, 8(2) (2000) 171-184.

[13] I.Ch. Paschalidis and Y. Liu, Pricing in Multiservice Loss Networks: Static Pricing, Aysmptotic Optimality, and Demand Substitution Effects, IEEE/ACM Transactions on Networking, 10(3) (2002) 425-438.

[14] E. Pinsky and A.E. Conway, Computational Algorithms for Blocking Probabilities in CircuitSwitched Networks, Annals of Operations Research, 35 (1992) 31-41.

[15] K.W. Ross, Multiservice Loss Models for Broadband Telecommunication Networks, SpringerVerlag, New York (1995). 
[16] K.W. Ross and D. Tsang, Algorithms to Determine Exact Blocking Probabilities for Multirate Tree Networks, IEEE Transactions on Communications, 38(8) (1990) 1266-1271.

[17] K.W. Ross and D.D. Yao, Monotonicity Properties of the Stochastic Knapsack, IEEE Transactions on Information Theory, 36(5) (1990) 1173-1179.

[18] W. Rudin, Principles of mathematical analysis, McGraw-Hill, New York (1976).

[19] A. Strzebonski, An Algorithm for Systems of Strong Polynomial Inequalities, The Mathematica Journal, 4(4) (1994) 74-77.

[20] R.W. Wolff and C. Wang, On the Convexity of Loss Probabilities, Journal of Applied Probability, 39 (2002) 402-406.

[21] S. Ziya, H. Ayhan and R.D. Foley, A Note on Optimal Pricing for Finite Capacity Queueing Systems with Multiple Customer Classes, Naval Research Logistics, 55 (2008) 412-418. 